\title{
Achieving better Authentication and Copyright protection Using DWT and SVD Based Watermarking Scheme
}

\author{
Anil Kumar Uppula ${ }^{1}$, Srinivasulu Tadisetty ${ }^{2}$ \\ ${ }^{1}$ PG Scholar, Dept of ECE, KU College of Engineering and Technology, Warangal, Telangana, (506132), INDIA \\ ${ }^{2}$ Professor, Dept of ECE, KU College of Engineering and Technology, Warangal, Telangana, (506132), INDIA \\ $\underline{\text { anil.uak555@gmail.com }}{ }^{1}, \underline{\text { drstadisetty@gmail.com }}{ }^{2}$
}

\begin{abstract}
Digital watermark has created ample solutions for securing hidden information in digital images. Incidental hacking and accidental hacking has increased in modern world which paves way for copying the legal information in illegal way. Although digital image processing has registered enormous growth in last few years but providing proper security to privacy information is still unresolved issue and watermarking is considered as accurate solution to all authentication and copyright protection issues in optimized way. DWT-SVD combinational watermarking scheme is proposed in this work for effective performance to provide good authentication and copyright protection and both techniques solves the technical issues related to embedding and extraction of information in three planes R (Red), G (Green) and B (Blue). Finally simulation results shows that the proposed method provides good performance over traditional state of art methods in effective way.
\end{abstract}

Keywords:SVD, DWT, Watermarking, Authentication, Copyright protection

\section{INTRODUCTION}

Implementation of digitalization in both image processing and signal processing domains have created revolutionary developments in various research fields like security, medicine, biometrics and genetics. The importance of security domain has increased in last two decades in enormous way. Technology is changing at fast pace and technologies related to security tend to provide authentication in equipped manner. Every second trillions of data bits are created in the information technology era and transmission of digital information has become so easy. Every technological invention has positive side as well a negative side and digitalization implementation in digital image processing domain too have both sides. Protecting privacy information has become concerned area in digital security systems and copying authenticated information in illegal has become easy.

Digital watermarking is approved as powerful tool for providing authentication and copyright protection. Digital watermarking has two steps (i) embedding and (ii) extraction, watermarking has been tested by various attacks and finally declared as most powerful security algorithm. Once digital content is watermarked then it creates difficulties for illegal hackers to detach the authenticated watermark. Digital
Watermarking too has some flaws and embedding large amount of information in image is critically unresolved issue.

\section{RELATED CONTENT}

\section{A. DISCRETE WAVELET TRANSFORM}

Discrete wavelet transform technique is a powerful tool used in modern applications for analysis in time, frequency and as well as spatial domain. Acquiring image statistics for analysis and image denoising is unresolved issues in digital image processing. Discrete wavelet transform have successfully replaced traditional algorithms and attains attention from international research standards because of its abilities to give statistics in less time and it denoise the noisy image using wavelet filters. The commonly used wavelet filters for image denoising are Haar Wavelet Filter, Daubechies Orthogonal Filters and Daubechies Bi-orthogonal Filters.

Human visual system (HVS) is basic parameter to determine the image quality while DWT is consider as most used tool in digital image processing and signal processing for analysis using decomposition. The decomposition is done to acquire statistical analysis based on 4 sub images namely LL, LH, $\mathrm{HL}$ and $\mathrm{HH}$ respectively. The four sub images represents approximation, vertical, detail and diagonal components of 
input image while LL is same as original image which represents low level frequencies. The multi-level decomposition is performed on LL image which can extend to till $8^{\text {th }}$ level respectively.

\section{B. SINGULAR VALUE DECOMPOSITION}

In general an image can be defined in two defined ways (i) statistical way (ii) perception way. By using SVD an image an image representation can be represented as positive scalar values i.e. matrix notation. SVD implementation on image can be factorized as $\mathrm{A}=\mathrm{USV}_{\mathrm{T}}, \mathrm{U}$ and $\mathrm{V}$ are orthogonal matrices where $U$ represents left singular matrices while $\mathrm{V}$ represents right singular vectors of respective image. $S$ term in SVD decomposition is defined as singular value in declining order. The data embedding in image is done in ease by using the SVD and the singular value is tend to use for embedding information i.e. watermark in efficient way.In digital watermarking schemes, SVD is used due to its main properties: 1) A small agitation added in the image, does not cause large variation in its singular values. 2) The singular value represents intrinsic algebraic image properties [4].

\section{LITERATURE SURVEY}

(1) An innovative approach for hiding data the data in digital images for providing robust copy right protection is presented by authors "W. L. Lin and W. H. Tsai" in the year 2004. The introduction of the boundary stream related to the data embedded is an idea in this approach and this boundary stream related to data are later embedded into mosaic image by detecting the respective boundary regions of preselected mosaic image in the Steganography application.

The copyright protection has been consistent concerned area in field of digital image processing and the respective method in this method helps to protect copyright protection by inserting the meaningful mosaic images into the paper copies. But this method fails when the attacks are done beyond limits.

(2) The generation of the mosaic images for protecting the copyright by using square shape tiles are introduced by $\mathrm{S}$. "C. Hung, T. Y. Liu and W. H. Tsai" in the year 2005. The main motto of this work is creating mosaic images to embed more data but the square shape data is not always done in all applications. The mosaic image generation in other shapes can increase embedded capacity in watermarking application. In this work the tiles used are not overlap with each others as occurs in conventional and the tiles orientation are always deterministic. The rotation and movement of tiles especially in the edges can pose problems of attacks. The work proposed in this method are tends for invisible watermarking which is used to providing robust copyright protection. This work leaves the future scope for creation of meaningful images in other shapes rather than square to increase the embedding capacity.

(3) A novel idea is proposed by "T. C. Wang and W. H. Tsai" in the year 2007, the proposed method mainly tends to introduce overlapping of mosaic images in horizontal and vertical tiles for the Steganography application. The creation of the hole has been concerned area in the past works and that is resolved in this paper.

Although the algorithm presented in this paper has good applications in real time scenario such as communications in secret way that is technically termed as covert communication. But this work in future can improve by introducing different shapes of tiles to overlap for increasing the data hiding capacity and to create the robustness against attacks.

\section{PROPOSED METHODOLOGY}

\section{A. EMBEDDING PROCESS}

Here we select a host image, primary watermark image and secondary watermark image of RGB image nature. We embed secondary watermark into the primary watermark. The resultant watermarked primary watermark is used as a watermark for the host image

Block diagram of watermark embedding process of the proposed method shown in Fig.4.1.

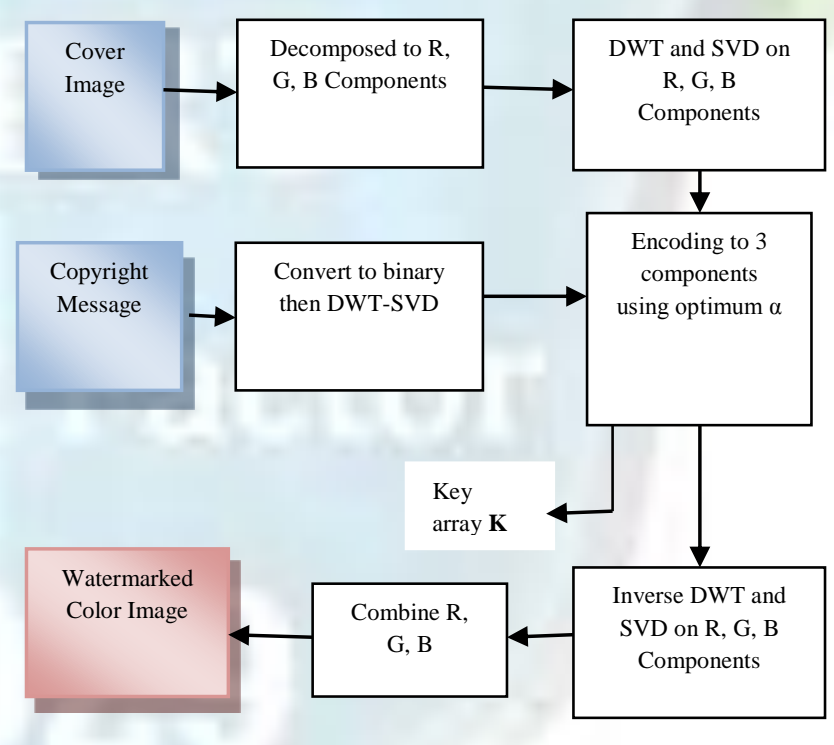

Fig.4. 1: Watermark embedding process of the proposed method

- In the first step the original image is decomposed into three (Red, Green and Blue) components by DWT transformation technique as shown below.

- This process decomposes each component value into 4 frequency sub-components (LL, HL, LH and $\mathrm{HH})$.

- Three levels of decomposition is applied separately to Red, Green and Blue components, as shown in Fig. 4.2. 
Anil Kumar Uppula and Dr.Srinivasulu Tadishetty," Achieving better Authentication and Copyright protection Using DWT and SVD Based Watermarking Scheme", International Journal of Computer Engineering In Research Trends, Volume 3, Issue 9, September2016, pp. 487-491

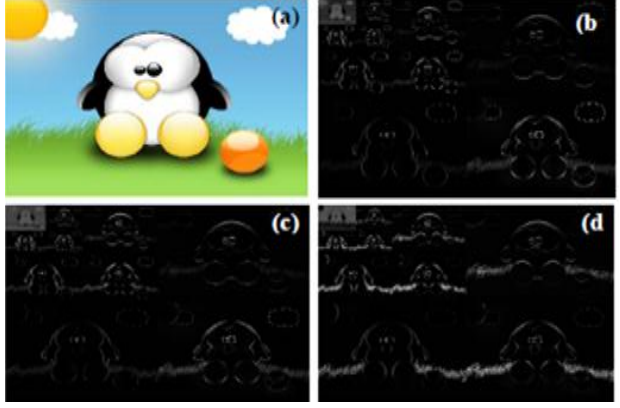

Fig.4.2: (a) original Image and 3rd level of DWT for (b) Red, (c) Green and (d) Blue

- The SVD decomposes each LH into U, V and S matrices.

- The singular values diagonal matrix (Sc) is used as a marking space in which the watermark information embeds.

- On the other hand, the watermark image is converted to a binary image, and then the DWT and SVD are applied to that binary image, extracting singular values diagonal matrix $(\mathrm{Sw})$.

- After then, the $\mathrm{Sw}$ is embedded with Sc using the following equation (1).

$\mathbf{S}_{\mathrm{c}}^{\prime}=\mathbf{S}_{\mathrm{c}}+\boldsymbol{\alpha} \cdot \mathbf{S}_{\mathrm{w}}$

Where $\alpha$ is a watermarking scaling factor.

In the proposed method, we use the optimum $\alpha$ value in the embedding and extracting processes for security purpose.

- The length of singular values diagonal matrix $(\mathrm{Sw})$ of a watermark image is less when compared to the length of singular values diagonal matrix $(\mathrm{Sc})$ of an original cover image for small watermark images.

- Thus, cover image's singular value matrix can be divided into different blocks.

- The number of blocks will be equal to the number of elements of singular values of the watermark image, and each singular value of watermark image can be added with a fixed position value of each block.

The key array (K) is used for storing the selected locations. After embedding, the inverse DWT and inverse SVD are applied for retrieving the R, G and B components. Finally, a watermarked color image is generated by combining all the three components.

\section{B. EXTRACTION PROCESS}

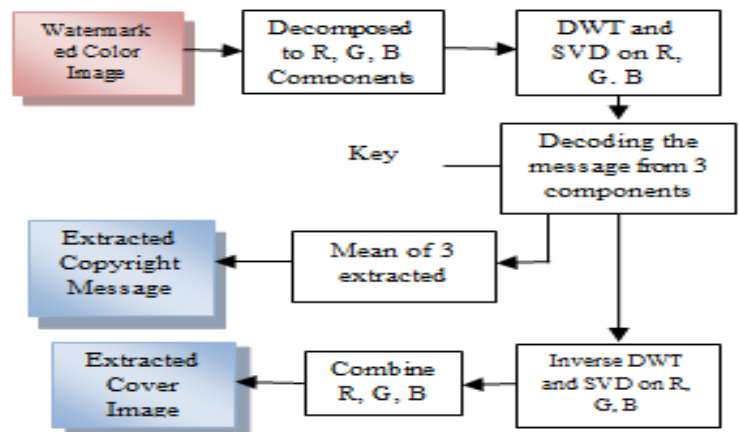

Fig.4.3: Watermark extraction process of the proposed method

Block diagram of extraction process of the proposed method shown in Fig. 4.3.

- In the extraction process, first decompose the watermarked color image into three $(\mathrm{R}, \mathrm{G}$ and $\mathrm{B})$ components.

- Three levels DWT is applied to each component and SVD decompose the LH sub-band of DWT. In the previous embedding process, watermark information is embedded in this singular diagonal value.

- The cover image and key array (K) are used for extracting the watermark from the diagonal matrix of each color component losslessly.

- After extracting the watermark image from three components, finally copyright message is extracted from the cover by calculating average of these color components.

\section{SIMULATION RESULTS}

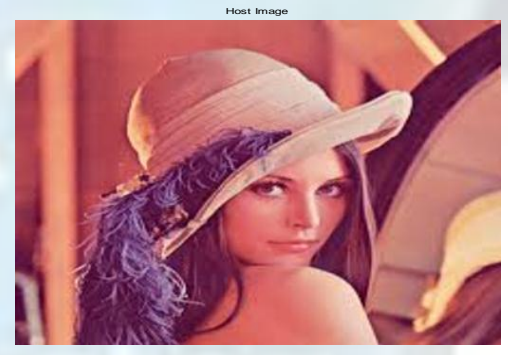

Fig.5.1: Host image

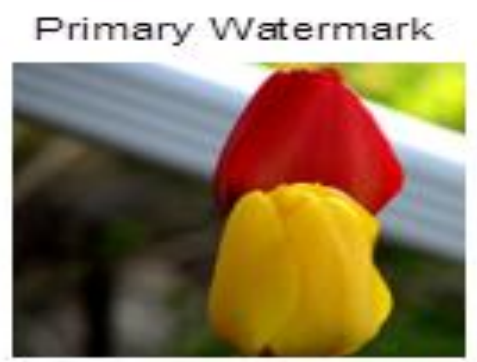

Fig.5.2: Primary watermarking

\section{Sec ondary Watemark}

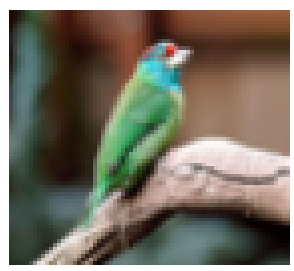

Fig.5.3: Secondary watermarking 
Anil Kumar Uppula and Dr.Srinivasulu Tadishetty," Achieving better Authentication and Copyright protection Using DWT and SVD Based Watermarking Scheme", International Journal of Computer Engineering In Research Trends, Volume 3, Issue 9, September2016, pp. 487-491

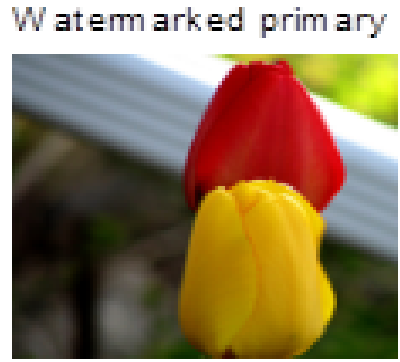

Fig.5.4: Watermarked primary

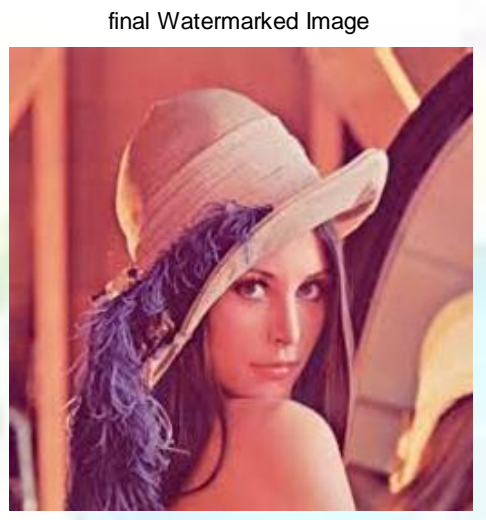

Fig.5.5: Final watermarked image

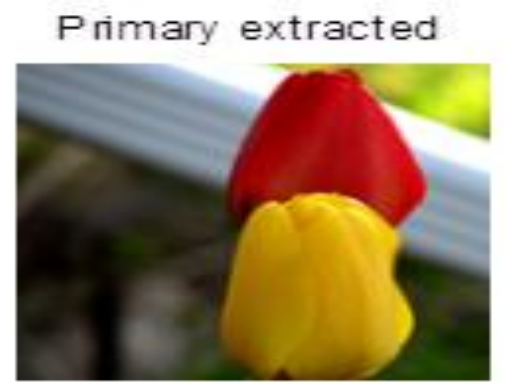

Fig.5.6: Primary extracted

\section{Secondary extracted}

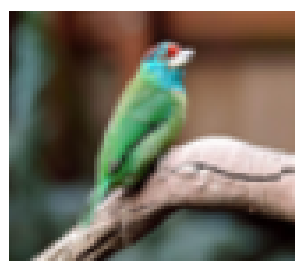

Fig.5.7: Secondary extracted

\section{IMPERCEPTIBILITY}

If we are going to apply watermark to any host image, the quality of the derived image get distorted. Imperceptibility of an image is nothing but is the perceived quality of an image that should not be distorted when watermark is introduced in an image.

To calculate the performance of proposed scheme, we have to use following parameters like PSNR (Peak Signal to Noise Ratio), MSE (Mean Square Error).

$$
\begin{gathered}
P S N R=10 \log _{10}(255 / M S E)^{2} d B \\
M S E=\sum_{i=0}^{n-1} \sum_{j=0}^{m-1} \frac{\left(a_{i j}-b_{i j}\right)^{2}}{n * m}(3)
\end{gathered}
$$

Where, $\mathrm{n} * \mathrm{~m}$ is the image size, $a_{i j}$ and $_{i j}$ are the corresponding pixels value of two image.

The obtained PSNR values for the two watermarks are:

$$
\begin{aligned}
& \text { PSNR_sec }=73.3629 \\
& \text { PSNR_pry }=98.9358
\end{aligned}
$$

\section{CONCLUSION}

DWT and DWT-SVD watermarking technique are applied to ensure the copyright protection and security of content or images and to increase the robustness of image. After applying both the watermarking techniques and comparing the values of PSNR at different values of scaling factor $\alpha$, it is concluded that the hybrid DWT-SVD technique is much better than DWT technique. In this paper, a comparative performance analysis by DWT-SVD based robust color image watermarking technique in RGB color space is presented. The SVD is an efficient tool for watermarking in the DWT domain.

\section{REFERENCES}

[1] Wang Hui-Qin; Zhao Min; , “A Blind Watermarking Algorithm for Color Image Based on Singular Value Quantization" 6th International Conference on Digital Content, Multimedia Technology and its Applications (IDC), 2010 ,PP 59 - 62,IEEE.

[2] Yan Xing and Jieqing Tan, A Color Watermarking Scheme Based on Block-SVD and Arnold Transformation, Second Workshop on Digital Media and its Application in Museum \& Heritages, PP 3 - 8,IEEE 2007.

[3] Jianyong Huang and Changsheng Yang, Image Digital Watermarking Algorithm Using Multiresolution Wavelet Transform, IEEE International Conference on Systems, Man and Cybernetics,PP 2977 - 2982 ,vol.3,IEEE 2004 .

[4] Chaokun Wang; Jianmin Wang; Ming Zhou; Guisheng Chen; Deyi Li; ATBaM: An Arnold Transform Based Method onWatermarking Relational Data, . International Conference on Multimedia and Ubiquitous Engineering, 2008. MUE 2008,PP 263 - 270,IEEE 2008

[5] Mabtoul, S.; Hassan, E.; Elhaj, I.; Aboutajdine, D.;" Robust color image watermarking based on singular value decomposition and Dual tree complex wavelet transform" 14th IEEE International Conference on Electronics, Circuits and Systems, 2007. ICECS 2007.,PP 534 - 537,IEEE 
Anil Kumar Uppula and Dr.Srinivasulu Tadishetty," Achieving better Authentication and Copyright protection Using DWT and SVD Based Watermarking Scheme", International Journal of Computer Engineering In Research Trends, Volume 3, Issue 9, September2016, pp. 487-491

[6] Deng Jianghua ,"Color Image Digital Watermarking Algorithm Based on Singular Value Decomposition", International Conference on Multimedia Information Networking and Security, 2009. MINES '09. PP 130 133,IEEE

[7] Wang Hui-qin; HaoJi-chao; Cui Fu-ming; Color Image Watermarking Algorithm Based on the Arnold Transform, 2010 International Conference on Communications and Mobile Computing,PP 66 - 69,IEEE 2010

[8] Liu Fang; Wang YuKai; “ Restoring Of The Watermarking Image In Arnold Scrambling”, 2nd
International Conference on Signal Processing Systems (ICSPS),PP V1-771 - V1-774,IEEE 2010

[9] Chih-Chin Lai; Cheng-Chih Tsai; Digital Image Watermarking Using Discrete Wavelet Transform and Singular Value Decomposition, IEEE TRANSACTIONS ON INSTRUMENTATION AND MEASUREMENT, VOL. 59, NO. 11, NOVEMBER 2010

[10]. Fanzhi Kong; YizhunPeng; "Color Image Watermarking Algorithm Based On HSI Color Space”, 2nd International Conference on Industrial and Information Systems (IIS), PP 464 - 467,IEEE 2010 